\title{
Implicit Pseudo-Runge-Kutta Processes
}

\author{
By
}

Masaharu NAKASHIMA*

\section{§1. Introduction}

The present paper is concerned with the numerical solution of the initial value problem;

$$
\left\{\begin{array}{l}
y^{\prime}=f(x, y) \\
y\left(x_{0}\right)=y_{0}
\end{array}\right.
$$

Of all computational one step methods for the numerical solution of this problem, the easiest formula is Euler's rule.

It is linear and explicit, but it is of low order.

Higher order methods have been achieved by sacrificing the linearity. These methods were first proposed by Runge [13] and subsequently developed by Heun [7] and Kutta [8].

Runge-Kutta methods need some functional evaluation at each step. And one looked for other methods to decrease the functional evaluation. These works were due to Ceschino, Kuntzman [4], Byrne, Lambert [1] and Rosen [12].

Now Byrne and Lambert [1] have defined 2-step Runge-Kutta methods and Costabiles [5] has proposed Pseudo-Runge Kutta methods. In [9], the present author has studied Pseudo-Runge Kutta methods and has proposed some new ones.

In [1], [5], [9] and [10], the methods are explicit forms, however, no implicit methods have been proposed as yet.

It is possible to consider implicit Pseudo-Runge Kutta methods, and in this paper, we shall give implicit Pseudo-Runge Kutta methods based on the methods developed in [10].

It will be seen that they are equivalent to certain implicit Runge-

Communicated by S. Hitotumatu, March 19, 1982.

* Department of Mathematics, Faculty of Science, Kagoshima University, Kagoshima 890, Japan. 
Kutta methods in some special cases.

Their advantage over implicit Runge-Kutta methods lies in the fact they are of less expensive in terms of the functional evaluations for given order.

Let $m(p)$ be the highest order which can be attained by a $p$-stage method. In [3], Butcher proved, on his method, the following results

$$
m(p)=2 p(p=1,2,3, \cdots),
$$

whereas our methods have

$$
m(p)=p+3 \quad(p=2,3) .
$$

In order to apply implicit Runge-Kutta methods, it is necessary, at each step, to solve non-linear equations by some means. By making clear the algorithm of our methods of $r$-stage, we observe that the number of non-linear equations is $r-1$.

Let $n(r)$ be the highest order that can be attained by non-linear equations of order $r$. Then by our methods

$$
n(r)=r+4 \quad(r=1,2) \text {, }
$$

and by Runge-Kutta methods

$$
n(r)=2 r(r=1,2, \cdots) \text {. }
$$

\section{§2. Derivation of the Methods}

We consider $p$-stage implicit Pseudo-Runge-Kutta methods:

$$
y_{n+1}=y_{n}+v\left(y_{n-1}-y_{n}\right)+h \Phi\left(x_{n-1}, x_{n}, y_{n-1}, y_{n} ; h\right) \text {, }
$$

where

$$
\begin{aligned}
& \Phi\left(x_{n-1}, x_{n}, y_{n-1}, y_{n} ; h\right)=\sum_{i=0}^{p} w_{i} k_{i}, \\
& k_{0}=f\left(x_{n-1}, y_{n-1}\right), k_{1}=f\left(x_{n}, y_{n}\right), \\
& k_{i}=f\left(x_{n}+a_{i} h,\left(1+b_{i}\right) y_{n}-b_{i} y_{n-1}+h \sum_{j=0}^{p} b_{i j} k_{j}\right), \\
& \quad(i=2,3, \cdots, p) \quad\left(0<a_{i} \leqq 1 ; i=2,3, \cdots, p\right) .
\end{aligned}
$$

In the above formula (2.1), the value $y_{n}$ is to be an approximation to the value $y\left(x_{n}\right)$ of the solution of (1.1) for $x_{n}=x_{0}+n h$. Coefficients $a_{i}, b_{i}(i=2,3, \cdots, p)$ and $b_{i j}(i=2,3, \cdots, p, j=0,1, \cdots, p)$ are real constants to be determined. 
We consider only two and three stage methods obtained by setting $p=2,3$ in (2.1). Throughout the paper, the coefficients are constrained by

$$
a_{i}=b_{i}+\sum_{j=0}^{p} b_{i j}(i=2,3, \cdots, p) .
$$

The functions $k_{i}(i \geqq 2)$ are no longer defined by explicitly but by the set of $p-1$ implicit equations. The special case $v=w_{0}=w_{1}=$ $b_{i}=b_{i 0}=0$ in (2.1) is implicit Runge-Kutta method. The derivation of the method is rather complicated. The treatement is similar as those in Butcher [3].

Let $D$ be the differential operator defined by

$$
D=\frac{\partial}{\partial x}+f\left(x_{n}, y_{n}\right) \frac{\partial}{\partial y},
$$

and put

$$
\begin{aligned}
& D^{i} f\left(x_{n}, y_{n}\right)=T^{i}(i=1,2, \cdots, 5), D^{i} f_{y}\left(x_{n}, y_{n}\right)=S^{i}(i=1,2,3), \\
& \left(D f_{y}\right)^{2}\left(x_{n}, y_{n}\right)=P,(D f)^{2}\left(x_{n}, y_{n}\right)=Q, D f_{y y}\left(x_{n}, y_{n}\right)=R, \\
& f_{y}\left(x_{n}, y_{n}\right)=f_{y}, f_{y y}\left(x_{n}, y_{n}\right)=f_{y y} .
\end{aligned}
$$

We also introduce an abbreviation,

$$
\sum=\sum_{i=2}^{3}, \quad \sum_{\boldsymbol{\ell}}=\sum_{\boldsymbol{\ell}=2}^{3} .
$$

We assume that the solutions of the functions $k_{i}(i \geqq 2)$ may be expressed in the form:

$$
k_{i}=f_{n}+\sum_{j=1}^{5} c_{i j} h^{j}+O\left(h^{6}\right),
$$

and

$$
y_{n-1}=y\left(x_{n-1}\right) .
$$

From (2.1), we have the following equation:

$$
\begin{aligned}
y_{n+1}= & y_{n}+h A_{1} k_{1}+h^{2} A_{2} T+\frac{1}{2 !} h^{3}\left(A_{3} f_{y} T+A_{4} T^{2}\right)+\frac{1}{3 !} h^{4}\left(B_{1} T^{3}+B_{2} f_{y} T^{2}\right. \\
& \left.+B_{3} f_{y}^{2} T+3 B_{4} S T\right)+\frac{1}{4 !} h^{5}\left(C_{1} T^{4}+6 C_{2} T S^{2}+4 C_{3} T^{2} S+3 C_{4} f_{y y} Q\right. \\
& \left.+C_{5} f_{y} T^{3}+C_{6} f_{y}^{2} T^{2}+C_{7} f_{y}^{3} T+C_{8} f_{y} T S\right)+\frac{1}{5 !} h^{6}\left(D_{1} T^{5}+D_{2} T S^{3}\right. \\
& +D_{3} T^{2} S^{2}+D_{4} T^{3} S+D_{5} f_{y y} T^{2} T+D_{6} Q R+D_{7} T P+D_{8} f_{y} T^{4}+D_{9} f_{y}^{2} T^{3} \\
& \left.+D_{10} f_{y}^{3} T^{2}+D_{11} f_{y}^{4} T+D_{12} f_{y y} f_{y} Q+D_{13} f_{y} T S^{2}+D_{14} f_{y}^{2} T S+D_{15} f_{y} T^{2} S\right)
\end{aligned}
$$


$+O\left(h^{7}\right)$.

The constants $\left\{A_{i}\right\},\left\{B_{i}\right\},\left\{C_{i}\right\}$ and $\left\{\mathrm{D}_{i}\right\}$ are

$$
\begin{aligned}
& A_{1}=-v+w_{0}+\sum w_{i}, \\
& A_{2}=\frac{v}{2}-w_{0}+\sum a_{i} w_{i}, \\
& A_{3}=\frac{-v}{3}+w_{0}+\sum p_{1 i} w_{i}, \\
& A_{4}=\frac{-v}{3}+w_{0}+\sum a_{i}^{2} w_{i}, \\
& B_{1}=\frac{v}{4}-w_{0}+\sum a_{i}^{3} w, \\
& B_{2}=\frac{v}{4}-w_{0}+\sum p_{2 i} w_{i}, \\
& B_{3}=\frac{v}{4}-w_{0}+\sum q_{2 i} w_{i}, \\
& B_{4}=\frac{v}{4}-w_{0}+\sum a_{i} p_{1 i} w_{i}, \\
& C_{1}=\frac{-v}{5}+w_{0}+\sum a_{i}^{4} w_{i}, \\
& C_{2}=\frac{-v}{5}+w_{0}+\sum a_{i}^{2} p_{1 i} w_{i}, \\
& C_{3}=\frac{-v}{5}+w_{0}+\sum a_{i} p_{2 i} w_{i}, \\
& C_{4}=\frac{-v}{5}+w_{0}+\sum p_{1 i}^{2} w_{i}, \\
& C_{5}=\frac{-v}{5}+w_{0}+\sum p_{3 i} w_{i}, \\
& C_{6}=\frac{-v}{5}+w_{0}+\sum g_{3 i} w_{i}, \\
& C_{7}=\frac{-v}{5}+w_{0}+\sum r_{3 i} w_{i}, \\
& C_{8}=\frac{-v}{5}+w_{0}+\sum q_{3 i} w_{i}, \\
& D_{1}=\frac{v}{6}-w_{0}+\sum a_{i}^{5} w_{i}, \\
& D_{2}=10\left(\frac{v}{6}-w_{0}+\sum a_{i}^{3} p_{1 i} w_{i}\right) \text {, } \\
& D_{3}=10\left(\frac{v}{6}-w_{0}+\sum a_{i}^{2} p_{2 i} w_{i}\right), \quad D_{4}=5\left(\frac{v}{6}-w_{0}+\sum a_{i} p_{3 i} w_{i}\right), \\
& D_{5}=10\left(\frac{v}{6}-w_{0}+\sum p_{1 i} p_{2 i} w_{i}\right), \quad D_{6}=15\left(\frac{v}{6}-w_{0}+\sum a_{i} p_{1 i}^{2} w_{i}\right) \text {, } \\
& D_{7}=15\left(\frac{v}{6}-w_{0}+\sum a_{i} q_{3 i} w_{i}\right), \quad D_{8}=3\left(\frac{v}{6}-w_{0}+\sum p_{4 i} w_{i}\right)+4 C_{3} \\
& D_{9}=\frac{v}{6}-w_{0}+\sum g_{4 i} w_{i} \\
& D_{10}=\frac{v}{6}-w_{0}+\sum r_{4 i} w_{i} \\
& D_{11}=\frac{v}{6}-w_{0}+\sum u_{1 i} w_{i} \\
& D_{12}=3\left(\frac{v}{6}-w_{0}+\sum h_{1 i} w_{i}\right)+10\left(\frac{v}{6}-w_{0}+\sum p_{1 \imath} q_{2 i} w_{i}\right), \\
& D_{13}=16\left(\frac{v}{6}-w_{0}+\sum q_{4 i} w_{i}\right), \quad D_{14}=7\left(\frac{v}{6}-w_{0}+\sum u_{2 i} w_{i}\right), \\
& D_{15}=4\left(\frac{v}{6}-w_{0}+\sum u_{3 i} w_{i}\right) \\
& p_{i j}=(i+1) !\left\{(-1)^{i} \frac{1}{(i+1) !} b_{j 0}+(-1)^{i} \frac{1}{i !} b_{j 1}+\frac{1}{i !} \sum_{\ell} a_{\ell}^{i} b_{j \ell}\right\} \text {, } \\
& q_{i j}=(i+1) !\left\{(-1)^{i} \frac{1}{(i+1) !} b_{j 0}+(-1)^{i} \frac{1}{i !} b_{j 1}+\frac{1}{i !} \sum_{\ell} a_{\ell}^{i-2} p_{1 \ell} b_{j \ell}\right\},
\end{aligned}
$$




$$
\begin{aligned}
& h_{1 j}=5 !\left\{\frac{1}{5 !} b_{j 0}+\frac{1}{4 !} b_{j 1}+\frac{1}{4 !} \sum_{\ell} p_{1 \ell}^{2} b_{j \ell}\right\}, \\
& g_{i j}=(i+1) !\left\{\frac{(-1)^{i}}{(i+1) !} b_{j 0}+\frac{1}{i !} b_{j 1}+\frac{1}{i !} \sum_{\ell} p_{i-1 \ell} b_{j \ell}\right\}, \\
& r_{i j}=(i+1) !\left\{\frac{(-1)^{i}}{(i+1) !} b_{j 0}+\frac{(-1)^{i}}{i !} b_{j 1}+\frac{1}{i !} \sum_{\ell} q_{i-1 \ell} b_{j \ell}\right\}, \\
& u_{1 j}=5 !\left\{\frac{1}{5 !} b_{j 0}+\frac{1}{4 !} b_{j 1}+\frac{1}{4 !} \sum_{\ell} r_{3 \ell} b_{\ell}\right\}, \\
& u_{2 j}=5 !\left\{\frac{7}{5 !} b_{j 0}+\frac{7}{4 !} b_{j 1}+\frac{1}{4 !}\left(4 \sum_{\ell} a_{\ell} g_{2 \ell} b_{2 \ell}+3 \sum_{\ell} p_{3 \ell} b_{2 \ell}\right)\right\}, \\
& u_{3}=5 !\left\{\frac{1}{5 !} b_{j 0}+\frac{1}{4 !} b_{j 1}+\frac{1}{4 !} \sum_{\ell=2}^{p} a_{\ell} p_{j 2 \ell} b_{j \ell}\right\} .
\end{aligned}
$$

The $p$-stage method (2.1) has order $p+3(p=2,3)$ if

$$
\begin{aligned}
& (-1)^{i} \frac{v}{i !}+(-1)^{i-1} \frac{w_{0}}{(i-1) !}+\sum_{j=2}^{r} \frac{a_{j}^{i-1}}{(i-1) !} w_{j}=\frac{1}{i !}, \\
& (-1)^{k-1} \frac{b_{j}}{k !}+(-1)^{k-1} \frac{b_{j 0}}{(k-1) !}+\sum_{\ell=2}^{r} \frac{a_{\ell}^{k-1} b_{j \ell}}{(k-1) !}=\frac{a_{j}^{k}}{k !}, \\
& (-1)^{p} \frac{v}{(p+3) !}+(-1)^{p-1} \frac{w_{0}}{(p+2) !}+\sum_{j=2}^{p}\left\{\frac{(-1)^{p+1}}{(p+2) !} b_{j}\right. \\
& \left.-\frac{(-1)^{p+1}}{(p+1) !} b_{j 0} \sum_{\ell=2}^{p} b_{j \ell}\left(\frac{(-1)^{p}}{(p+1) !} b_{\ell}+(-1)^{p} \frac{b_{\ell 0}}{p !}+\frac{1}{p !} \sum_{r=2}^{p} a_{r} b_{\ell r}\right)\right\} w_{j} \\
& =\frac{1}{(p+3) !}
\end{aligned}
$$

$(i=1,2, \ldots, p+3, j=2,3, \ldots, p, k=2,3, \ldots, p+1),\left(a_{i} \neq a_{j}\right.$ for $i \neq j)$.

The case $p=2$ in (2.4) gives the following values for the constants in (2.1). It is of order 5 ,

$$
\begin{aligned}
& v=77-12 c, w_{0}=\frac{1}{4}(45-7 c), w_{1}=\frac{1}{2}(33-5 c), \\
& w_{2}=\frac{1}{4}(201-31 c), a_{2}=\frac{1}{10}(1+c), b_{2}=\frac{1}{250}(-413+47 c), \\
& b_{20}=\frac{1}{125}(37-3 c), b_{21}=\frac{1}{250}(139+9 c), b_{22}=\frac{1}{10}(9-c), \\
& \quad(c=\sqrt{41}) .
\end{aligned}
$$

The case $p=3$ in (2.4) gives the following values for the constants in (2.1). It is of order 6, 


$$
\begin{aligned}
& a_{3}= \begin{cases}-\left(-a_{2}^{2}+\frac{2}{3} a_{2}+\frac{1}{3}\right)+\sqrt{d_{1}} & \left(0<a_{2} \leqq(1+\sqrt{41}) / 10\right) \\
\frac{-\left(5 a_{2}^{2}-a_{2}-2\right)}{-\left(-a_{2}^{2}+\frac{2}{3} a_{2}+\frac{1}{3}\right)-\sqrt{d_{1}}} & \left((1+\sqrt{41}) / 10<a_{2} \leqq 1\right),\end{cases} \\
& w_{3}=\frac{\left(5 a_{2}+3\right)\left(\frac{5}{6} a_{2}-\frac{7}{12}\right)+5\left(2 a_{2}+1\right)\left(\frac{7}{12} a_{2}-\frac{9}{20}\right)}{a_{3}\left(1+a_{3}\right)\left(a_{2}-a_{3}\right)\left\{\left(5 a_{2}+3\right)+5 a_{3}\left(2 a_{2}+1\right)\right\}}, \\
& v= \begin{cases}\left.a_{3}^{2}\left(1+a_{3}\right)\left(a_{2}-a_{3}\right) w_{3}-\left(\frac{7}{12} a_{2}-\frac{9}{20}\right)\right\}\left(\frac{1}{12} a_{2}+\frac{1}{20}\right)^{-1}, \\
w_{2}=\frac{5-v-6 a_{3}\left(1+a_{3}\right) w_{3}}{6 a_{2}\left(1+a_{2}\right)}, w_{0}=\frac{1}{2}(v-1)+\sum a_{i} w_{i},\end{cases} \\
& w_{1}=1-\left(w_{0}+\sum w_{i}-v\right), \quad(i=2,3), \\
& b_{i 2}=\frac{\left\{a_{i}\left(a_{i}+1\right)\right\}^{2}-2 a_{3}\left(2 a_{3}^{2}+3 a_{3}+1\right) b_{i 3},}{2 a_{2}\left(2 a_{2}^{2}+3 a_{2}+1\right)}, \\
& b_{i}=6\left(a_{2}\left(a_{2}+1\right) b_{i 2}+a_{3}\left(1+a_{3}\right) b_{i 3}\right)-2 a_{i}^{3}-3 a_{i}^{2}, \\
& b_{i 0}=\frac{1}{2}\left(-b_{i}+2 \sum_{j=2}^{3} a_{j} b_{i j}-a_{i}^{2}\right), \\
& b_{23}=\frac{1}{30 d_{2} w_{2}}\left\{1-v-6 w_{0}+6 w_{3}\left(b_{3}+5 b_{30}+5 a_{2}^{4} b_{32}+a_{3}^{4} b_{33}\right)-d_{3}\right\}, \\
& b_{i 1}=a_{i}-\left(b_{i}+b_{i 0}+b_{i 1}+b_{i 2}\right)
\end{aligned}
$$

where

$$
\begin{aligned}
& d_{1}=\left(-a_{2}^{2}+\frac{2}{3} a_{2}+\frac{1}{3}\right)^{2}+4\left(5 a_{2}^{2}-a_{2}-2\right)\left(2 a_{2}^{2}-\frac{1}{3} a_{2}-1\right), \\
& d_{2}=\frac{1}{5}\left\{a_{3}\left(5 a_{3}^{3}-9 a_{3}-4\right)-\frac{5 a_{2}^{3}-9 a_{2}-4}{2 a_{2}^{2}+3 a_{2}+1} a_{3}\left(2 a_{3}^{2}+3 a_{3}+1\right)\right\}, \\
& d_{3}=\frac{1}{5}\left\{a_{2}^{2}\left(3 a_{2}+2\right)+\frac{5 a_{2}^{3}-9 a_{2}-4}{4 a_{2}^{2}+6 a_{2}+2}\left(a_{2}\left(a_{2}+1\right)\right)^{2}\right\}
\end{aligned}
$$

\section{§3. Convergence of Our Methods}

In order to apply the method (2.1), it is necessary to solve, at each step, non-linear algebraic equations. We seek a solution by an inner iterative procedure and prove the convergence of the method (2. 1).

Butcher [3] studied $p$-stage implicit Runge-Kutta method and gave the iterations 


$$
k_{r}^{(N+1)}=f\left(x+a_{i} h, y+h \sum_{j=1}^{r-1} b_{i j} k_{j}^{(N+1)}+h \sum_{j=r}^{p} b_{i j} k_{j}^{(N)}\right) \quad(i=1,2, \ldots, p),
$$

where $k_{r}^{(N)}$ denotes the number after $N$-times iteration for the implicit non-linear equations of $k_{r}$. Similary, we set the iteration in (2.1) by

(3. 1) $k_{i}^{(N+1)}=f\left(x_{n}+a_{i} h,\left(1+b_{i}\right) y_{n}-b_{i} y_{n-1}+h \sum_{j=0}^{i-1} b_{i j} k_{j}^{(N+1)}+h \sum_{j=i}^{p} b_{i j} k_{j}^{(N)}\right)$.

Then we have the following Theorem.

Theorem. The iteration process (3.1) converges provided

$$
h \leqq 1 /\left\{L\left(U_{1}+U_{2}\right)\right\},
$$

where $L$ is the Lipshitz constant of $f(x, y)$ with respect to $y$ and

$$
\begin{aligned}
& U_{1}=\max \left\{\sum_{i=1}^{p}\left|b_{2 i}\right|, \quad \sum_{i=1}^{p}\left|b_{3 i}\right|\right\}, \\
& U_{2}=\max \left\{\sum_{i=1}^{p}\left|b_{i 2}\right|, \quad \sum_{i=1}^{p}\left|b_{i 3}\right|\right\} .
\end{aligned}
$$

The proof is done in a similar way as in Butcher [3] and we will omit it.

\section{§4. Stability Analysis}

In this section, we discuss the stability of our methods. We apply our method (2.1) with $p=2$ to the test equation $y^{\prime}=\lambda y$ which yields

$$
\begin{aligned}
V_{1} y_{n+1} & +V_{2} y_{n}+V_{3} y_{n-1}=0, \\
V_{1}= & 1-\frac{1}{10}(9-\sqrt{41}) \hat{h}, \\
V_{2}= & (-76+12 \sqrt{41})+(-5875.75+926.25 \sqrt{41}) \hat{h} \\
& +(2286.775-361.225 \sqrt{41}) \hat{h}^{2}, \\
V_{3}= & 77-12 \sqrt{41}+(4642.75-733.25 \sqrt{41}) \hat{h} \\
& +(11.25-1.75 \sqrt{41}) \hat{h}^{2} \quad(\hat{h}=\lambda h) .
\end{aligned}
$$

The condition for the stability is that the roots of characteristic equation of this difference equation are all of absolute value less than 1. This yields that, if $\lambda$ is negative real, the stability bound is

$$
-2.9 \leqq \hat{h} \leqq 0 \text {, }
$$

if $\lambda$ is complex, the stability region shown in Figure (1). 
Let us consider the method (2.1) with $p=3$. In that case we have

$$
y_{n+1}-\widetilde{V}_{1} y_{n}-\widetilde{V}_{2} y_{n-1}=0
$$

where

$$
\begin{aligned}
\tilde{V}_{1}= & 1-v+\left(e_{11}+w_{1}\right) \hat{h}+e_{12} \hat{h}^{2}+e_{13} \hat{h}^{3}, \\
\tilde{V}_{2}= & -v+\left(e_{21}-w_{0}\right) \hat{h}+e_{22} \hat{h}^{2}+e_{23} \hat{h}^{3}, \\
e_{11}= & \left\{\left(1+b_{2}\right) w_{2}+\left(1+b_{3}\right) w_{3}\right\} / L, \\
e_{12}= & \left\{\left(b_{21}-b_{33}\left(1+b_{2}\right)+b_{23}\left(1+b_{3}\right)\right) w_{2}+\left(b_{31}+b_{32}\left(1+b_{2}\right)\right.\right. \\
& \left.\left.-b_{22}\left(1+b_{3}\right)\right) w_{3}\right\} / L, \\
e_{13}= & \left\{\left(-b_{21} b_{33}+b_{23} b_{31}\right) w_{2}+\left(-b_{22} b_{31}+b_{21} b_{32}\right) w_{3}\right\} / L, \\
e_{21}= & -\left\{\left(b_{2} w_{2}+b_{3} w_{3}\right)\right\} / L, \\
e_{22}= & \left\{\left(b_{20}+b_{2} b_{33}-b_{23} b_{3}\right) w_{2}+\left(b_{30}+b_{22} b_{3}-b_{2} b_{32}\right) w_{3}\right\} / L, \\
e_{23}= & \left\{\left(-b_{20} b_{33}+b_{23} b_{30}\right) w_{0}+\left(-b_{22} b_{30}+b_{20} b_{32}\right) w_{3}\right\} / L, \\
L= & -\left\{1-\hat{h}\left(b_{22}+b_{33}\right)+\hat{h}^{2}\left(b_{22} b_{33}-b_{23} b_{32}\right)\right\} .
\end{aligned}
$$

Let us consider some specific algorithms in the formula (2.6)。 If we set

$$
b_{33}=-\frac{w_{2}\left(Z_{4}+Z_{5} Z_{10}\right)}{w_{2} Z_{6}-w_{3} Z_{5}},
$$

where

$$
\begin{aligned}
Z_{1}= & \frac{\left(a_{2}\left(a_{2}+1\right)\right)^{2}}{2 a_{2}\left(2 a_{2}^{2}+3 a_{2}+1\right)}, \\
Z_{2}= & \frac{\left(-2 a_{3}\left(2 a_{3}^{2}+3 a_{3}+1\right)\right)}{\left(2 a_{2}\left(2 a_{2}^{2}+3 a_{2}+1\right)\right)}, \\
Z_{3}= & \frac{\left(a_{3}\left(a_{3}+1\right)\right)^{2}}{\left(2 a_{2}\left(2 a_{2}^{2}+3 a_{2}+1\right)\right)}, \\
Z_{4}= & \left(a_{2}^{3}+a_{2}^{2}\right) Z_{3}-\left(a_{3}^{3}+a_{3}^{2}\right) Z_{1}, \\
Z_{5}= & \left\{\left(-2 a_{3}-3 a_{3}^{2}\right) Z_{3}-\left(a_{3}^{3}+a_{3}^{2}\right) Z_{2}\right\} w_{3}+ \\
& \left\{\left(-2 a_{2}-3 a_{2}^{2}\right) Z_{3}-2 a_{3}-3 a_{3}^{2}\right\} w_{2}, \\
Z_{6}= & \left\{\left(a_{2}^{3}+a_{2}^{2}\right) Z_{2}+\left(2 a_{3}+3 a_{3}^{2}\right) Z_{1}\right\} w_{3}-\left\{\left(-2 a_{2}-3 a_{2}^{2}\right) Z_{1}+\right. \\
Z_{7}= & \left(a_{2}^{4}-1.8 a_{2}^{2}-0.8 a_{2}\right) Z_{1}+0.2\left(3 a_{2}^{3}+2 a_{2}^{2}\right), \\
Z_{8}= & \left(a_{3}^{4}-1.8 a_{3}^{2}-0.8 a_{3}\right)-\frac{\left(a_{2}^{3}-1.8 a_{2}^{2}-0.8 a_{2}\right) a_{2} Z_{2}}{a_{2}}, \\
Z_{9}= & \left(a_{2}^{4}-1.8 a_{2}^{2}-0.8 a_{2}\right) Z_{3}+0.2\left(3 a_{3}^{3}+2 a_{3}^{2}\right), \\
Z_{10}= & \frac{1}{Z_{8} w_{2}}\left(\frac{1}{30}-\frac{v}{30}+\frac{w_{0}}{5}-Z_{9} w_{3}\right)-Z_{7} .
\end{aligned}
$$

Then we have 


$$
e_{23}=0 \text {. }
$$

If we set, in addition to the condition (4.2),

$$
a_{2}=0.98 \text {, }
$$

then we have the following stability bound

$$
-55 \leqq \hat{h} \leqq 0 \text {. }
$$

The stability region is shown in Figure (2).

If we set, in addition to the condition (4.2),

$$
a_{2}=0.57 \text {, }
$$

then we have the following stability bound

$$
-67 \leqq \hat{h} \leqq 0
$$

The stability region is shown in Figure (3).

Next we consider the case where the coefficient $b_{33}$ varies.

$$
\begin{aligned}
a_{2}= & 1, \\
b_{33}= & \{81(8 \sqrt{3}-14.89)-27(503.29-272 \sqrt{3}) \overline{\bar{h}}-9(2254.53 \\
& \left.-1254.71 \sqrt{3}) \overline{\bar{h}}^{2}-(352.8 \sqrt{3}-334.8) \overline{\bar{h}}^{3}\right\} /\{27(33.11 \sqrt{3} \\
& -65.34) \overline{\bar{h}}+9(937.2 \sqrt{33}-1861.86) \bar{h}^{2}-(475.2 \sqrt{3} \\
& \left.+118.8) \overline{h^{3}}\right\},
\end{aligned}
$$

where

$$
\begin{aligned}
& \hat{h}=a+b i, \\
& \bar{h}=\operatorname{sgn}(a) \sqrt{a^{2}+b^{2}} .
\end{aligned}
$$

In this case, we have the following characteristic roots:

$$
\begin{aligned}
& \rho_{1}=0.9, \\
& \rho_{2}=-\left\{\frac{s_{1} t_{1}+s_{2} t_{2}}{t_{1}^{2}+t_{2}^{2}}+a w_{0}\right\}-\left\{\frac{s_{2} t_{1}-s_{1} t_{2}}{t_{1}^{2}+t_{2}^{2}}+b w_{0}\right\},
\end{aligned}
$$

with

$$
\begin{aligned}
& s_{1}=(-128+30 \sqrt{3}) / 11+e_{22}\left(a^{2}-b^{2}\right)+e_{21} a+e_{23}\left(a\left(a^{2}-b^{2}\right)-2 a b^{2}\right), \\
& s_{2}=e_{21} b+2 e_{22} a b+e_{23}\left(b\left(a^{2}-b^{2}\right)+2 a^{2} b\right), \\
& t_{1}=1-\left(b_{22}+b_{33}\right) a+\left(b_{22} b_{33}-b_{23} b_{32}\right)\left(a^{2}-b^{2}\right), \\
& t_{2}=-\left(b_{22}+b_{33}\right)+2\left(b_{22} b_{33}-b_{23} b_{32}\right) a b .
\end{aligned}
$$

Similary, we look for the stability region numerically, which is contained in the region:

$$
D=\{(a, b):-280 \leqq a \leqq 0, \quad|b| \leqq 30\} .
$$


The stability region is shown in Figure (4). In the case (4.3), we have $b_{33} \longrightarrow \infty$ as $\bar{h} \longrightarrow 0$, however the iterations of function $k_{i}$ are convergent.

The stability intervals for $R-K$, Lawson's method (explicit $R-K$ type of order 5) and Huta's method (explicit $R-K$ type of order 6) are shown in Table (1). The stability regions for these methods are shown in Figure (5).

The stability intervals for $R-K 4$, Lawson's method and Huta's method.

$\begin{array}{lc}\text { Method } & \text { interval of stability } \\ R-K 4 & (-2.7,0) \\ \text { Lawson } & (-5.7,0) \\ \text { Hǔta } & (-3.7,0)\end{array}$

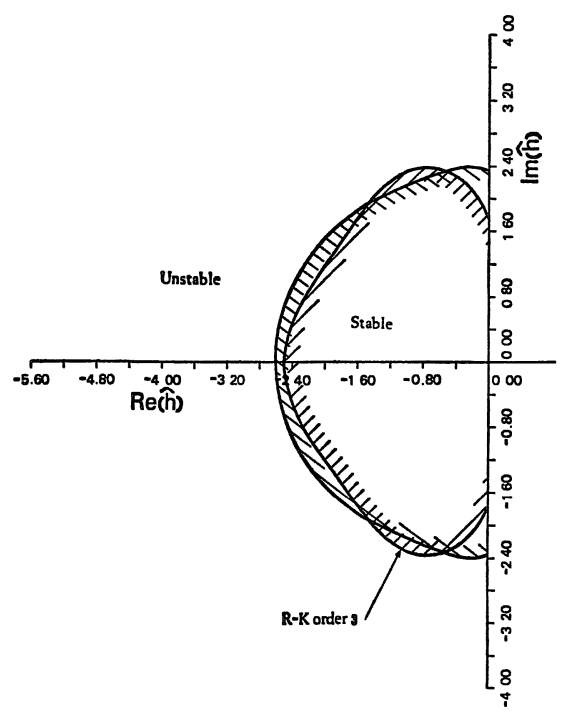

Figure (1). Stability region for the method (2.1) with $p=2$, whose coefficients are given by $(2.5)$. $(\operatorname{Re}(\lambda) \leqq 0)$. 


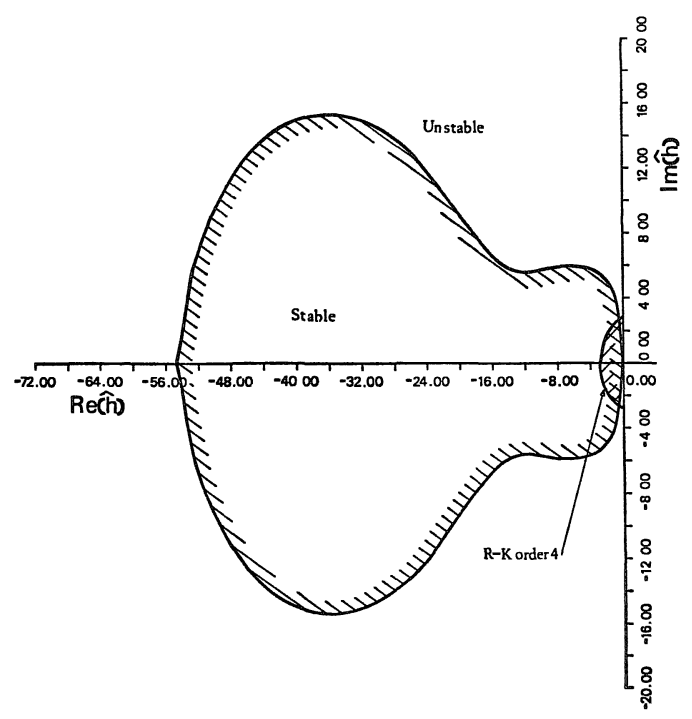

Figure (2). Stability region for the method (2.1) with $p=3$, whose coefficients are given by (2.6), (4.2) and $a_{2}=0.98$. $(\operatorname{Re}(\lambda) \leqq 0)$.

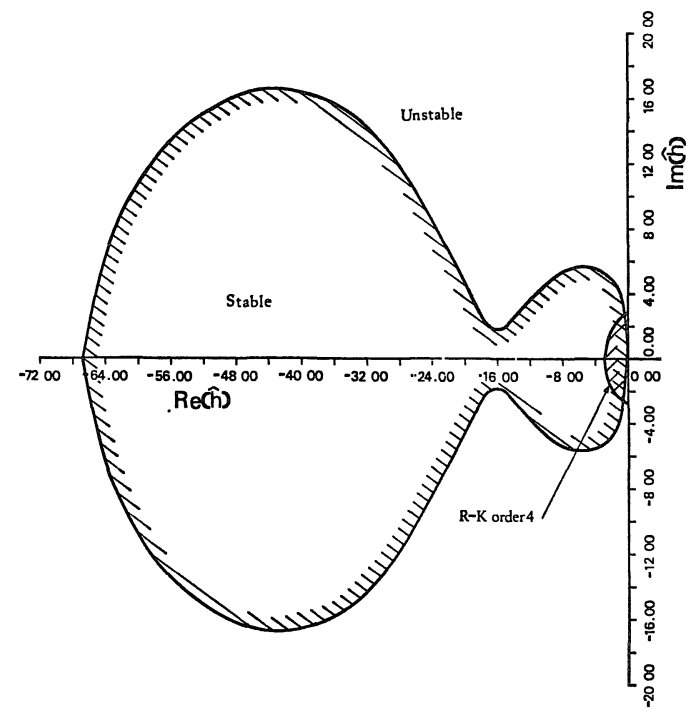

Figure (3). Stability region for the method (2.1) with $p=3$, whose coefficients are given by (2.6), (4.2) and $a_{2}=0.57 .(\operatorname{Re}(\lambda) \leqq 0)$. 


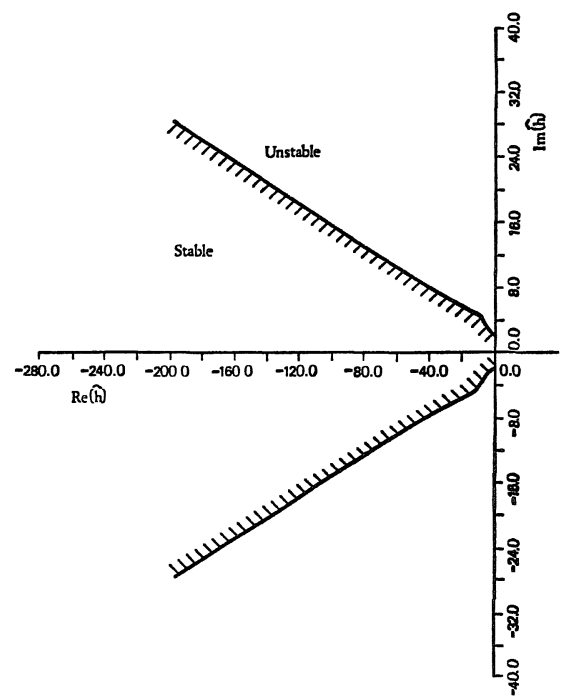

Figure (4). Stability region for the method (2.1) with $p=3$, whose coefficients are given by (2.6) and (4.3). $(\operatorname{Re}(\lambda) \leqq 0)$.

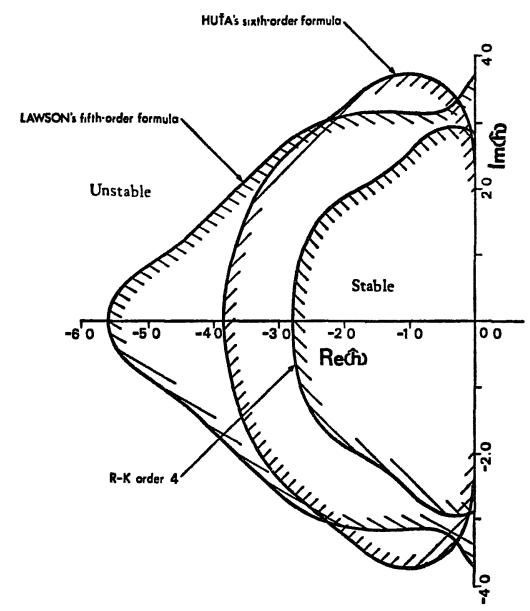

Figure (5). Stability region for $R-K$ method (order 4), Lawson's method (order 5) and Huta's method (order 6). $(\operatorname{Re}(\lambda) \leqq 0)$.

\section{§5. Computational Results}

In Table I and II, we present numerical results for the following initial value problems.

$$
\mathrm{I}: y^{\prime}=(y-x y) / x, y(1)=e^{-1}, y(x)=x e^{-x},
$$


II $: y^{\prime}=-y^{2}\left(2 e^{x}-1\right), y(0)=1, y(x)=1 /\left(2 e^{x}-x-1\right)$,

III : $\left\{\begin{array}{l}y^{\prime}=-y+z, y(0)=1, y(x)=(1+x) e^{-2 x} \\ z^{\prime}=-y-3 z, z(0)=0, z(x)=-x e^{-2 x}\end{array}\right.$

IV : $\left\{\begin{array}{l}y^{\prime}=-y+3 z-8 x-9, y(0)=6, y(x)=3 e^{x}+e^{-4 x}+x+2 \\ z^{\prime}=2(y-z)+4 x+7, z(0)=5, z(x)=2 e^{x}-e^{-4 x}+3 x+4\end{array}\right.$

Computations are done in double precision arithmetic on FACOM M-200 of Kyushu University.

In Table $I$, the value $y_{1}$ necessary for the evaluations using the formula (2.4) is computed by Runge-Kutta methods of order 4 and in Table II, the value $y_{1}$ necessary for the evaluations using the formula (4.1), (4.2) is computed by Huta's method of order 6 .

In order to start the caluculations at $\left(x_{n}, y_{n}\right)$, we need to solve the implicit functions $k_{i}(i \geqq 2)$. We first caluculate the predicted value for the functions by $k_{0}=f\left(x_{n}, y_{n}\right)$.

\section{Table I}

Error for the solutions to the Problems I, II, III, and IV. Comparison of errors incurred by using the implicit Runge-Kutta method of order 4 (Im $R-K 4$ ), Lawson's method (explicit $R-K$ type of order 5) and the method (2.1) with the coefficients (2.5) (order 5).

Problem I. $h=1 / 2^{4}, \mathrm{M}=5$ ( $\mathrm{M}$ : number of iterations),

\begin{tabular}{|c|c|c|c|}
\hline \multirow{3}{*}{$x$} & Lawson's Method & $\operatorname{Im} R-K 4$ & $\begin{array}{c}\text { method }(2.1) \\
\text { with } \quad(2.5)\end{array}$ \\
\hline & \multicolumn{2}{|c|}{ function evaluations/step } & \\
\hline & 6 & $11(1+2 \times M)$ & $6(1+1 \times M)$ \\
\hline 2 & $-0.9970 \mathrm{E}-10$ & $0.6963 \mathrm{E}-08$ & $-0.1442 \mathrm{E}-08$ \\
\hline 5 & $-0.7074 \mathrm{E}-11$ & $0.6034 \mathrm{E}-09$ & $-0.1408 \mathrm{E}-08$ \\
\hline 9 & $0.7337 \mathrm{E}-12$ & $0.2277 \mathrm{E}-10$ & $-0.6463 \mathrm{E}-11$ \\
\hline 13 & $0.5673 \mathrm{E}-13$ & $0.2142 \mathrm{E}-11$ & $-0.2671 \mathrm{E}-12$ \\
\hline
\end{tabular}

Problem II. $h=1 / 2^{4}, M=5$.

\begin{tabular}{|c|c|c|c|}
\hline$x$ & Lawson's Method & $\operatorname{Im} R-K 4$ & $\begin{array}{c}\text { method }(2.1) \\
\text { with }\end{array}$ \\
\hline 1 & $0.4180 \mathrm{E}-08$ & $-0.1528 \mathrm{E}-07$ & $0.1082 \mathrm{E}-07$ \\
\hline 4 & $0.1169 \mathrm{E}-09$ & $-0.4188 \mathrm{E}-10$ & $-0.2092 \mathrm{E}-12$ \\
\hline 8 & $0.1375 \mathrm{E}-11$ & $-0.4188 \mathrm{E}-10$ & $-0.2092 \mathrm{E}-12$ \\
\hline 12 & $0.2442 \mathrm{E}-13$ & $-0.1026 \mathrm{E}-11$ & $-0.2423 \mathrm{E}-13$ \\
\hline
\end{tabular}



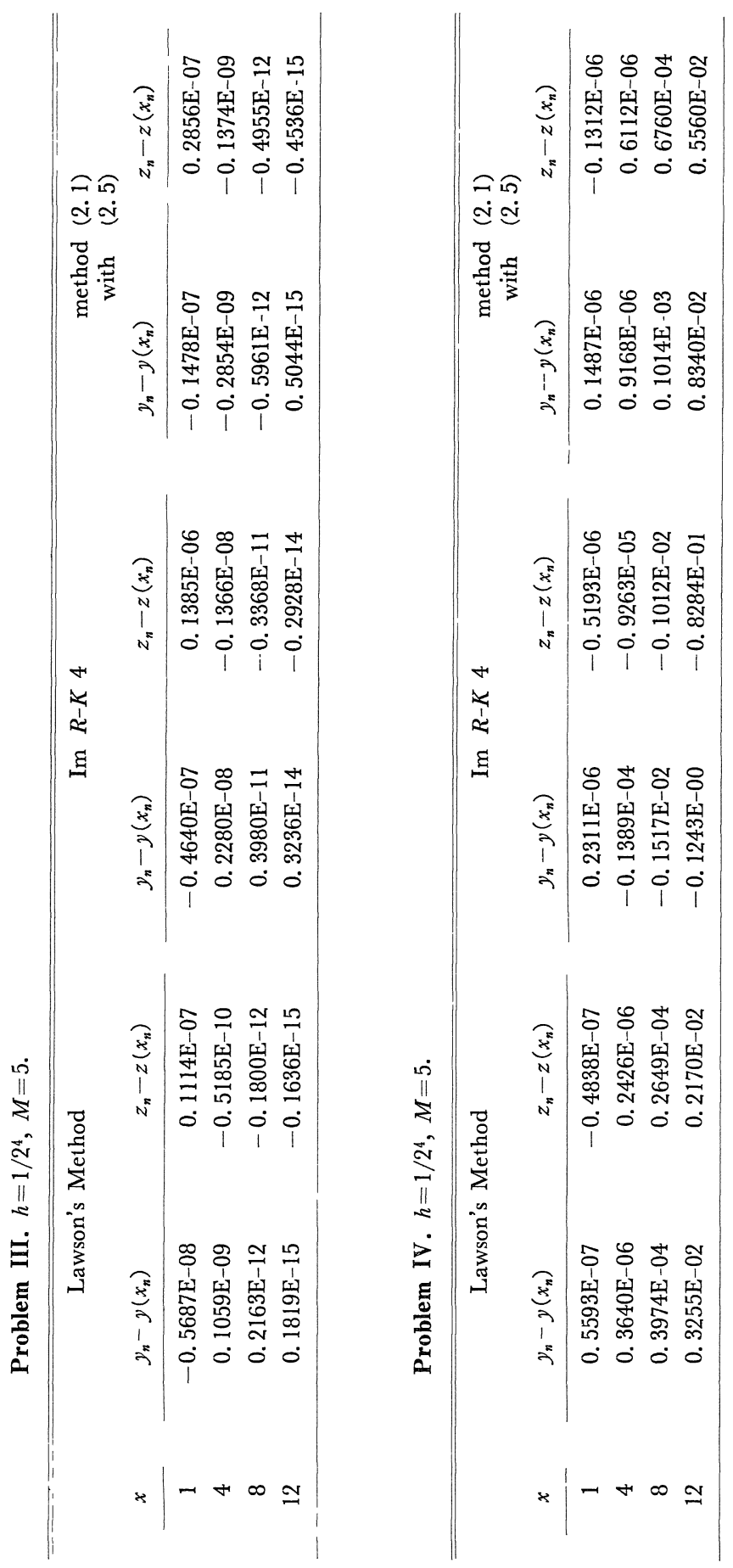

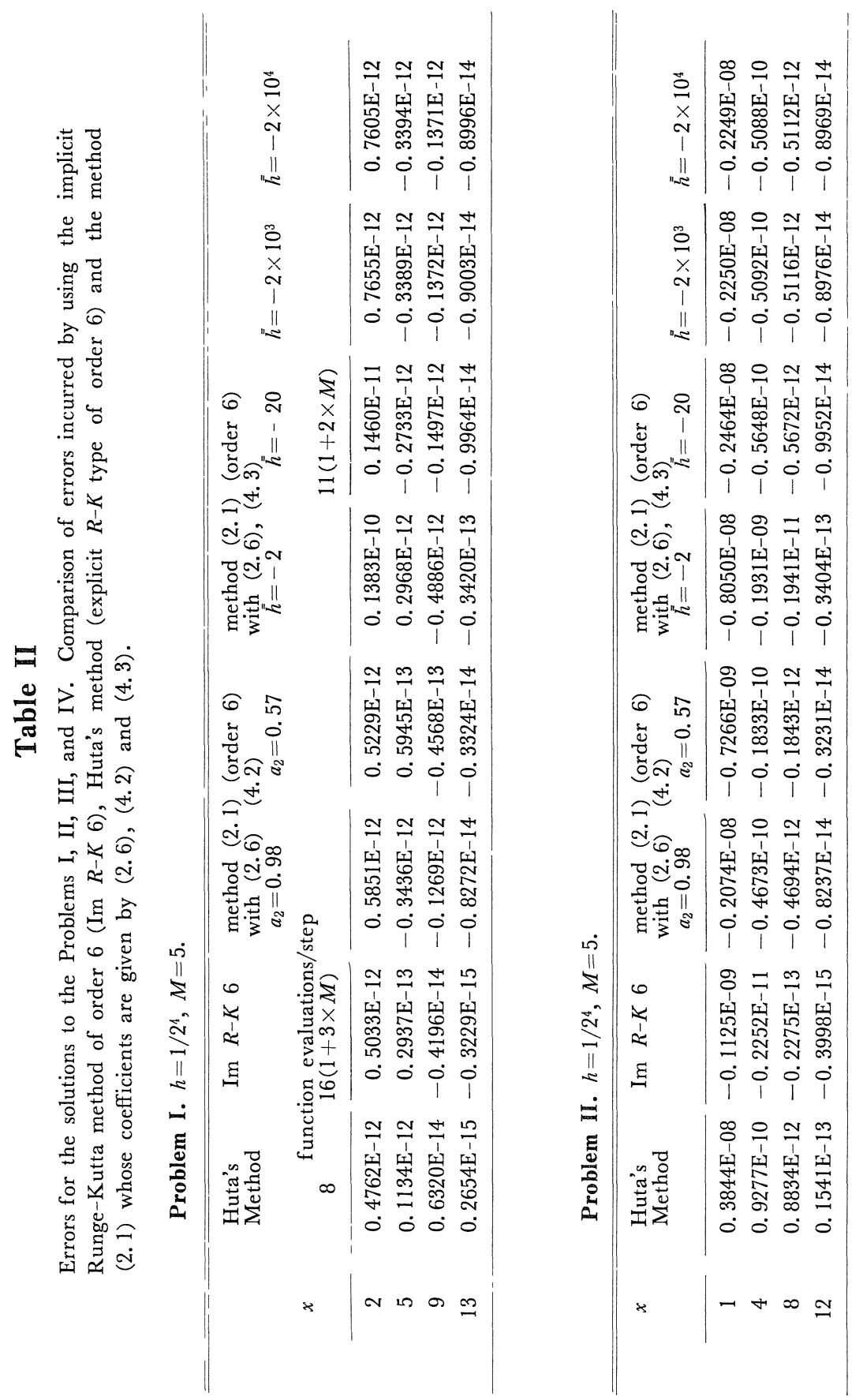

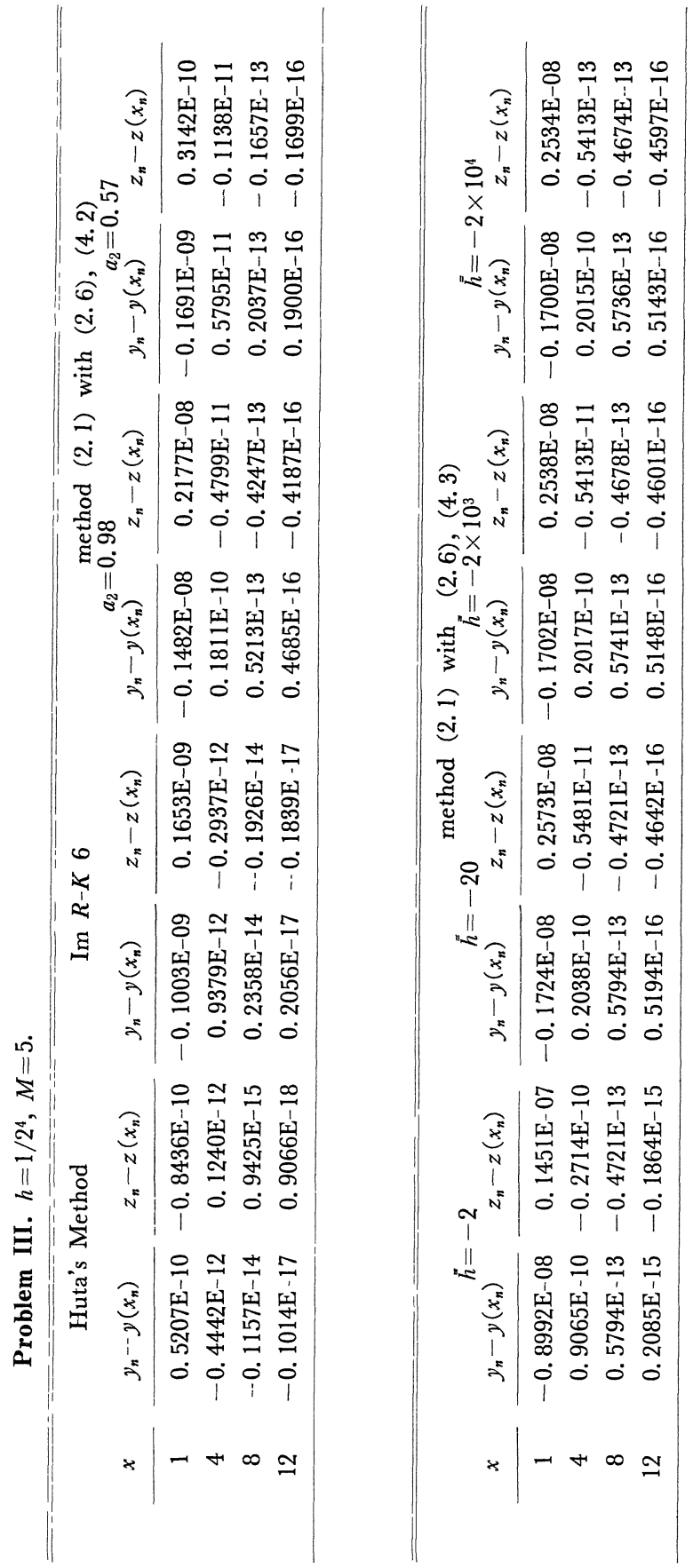

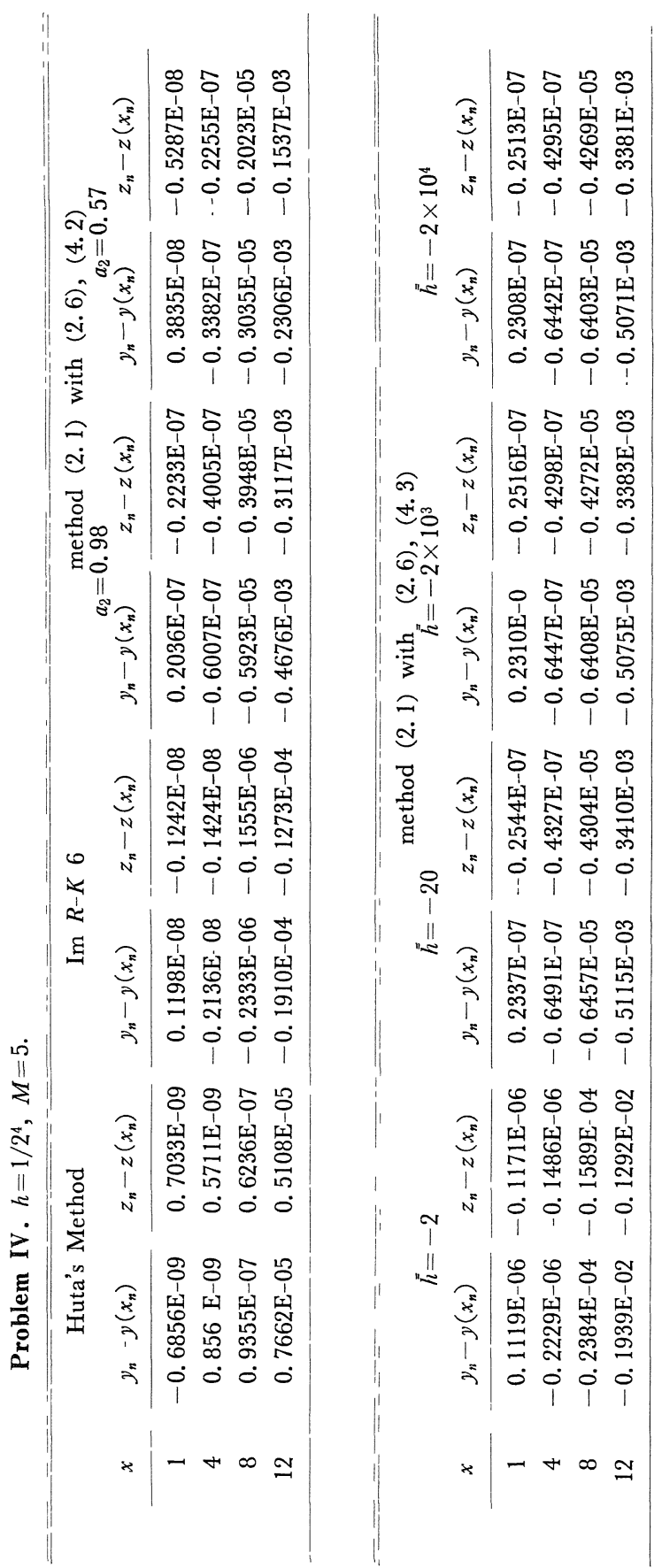


\section{Acknowledgements}

The author wishes to express his hearty thanks to Prof. M. Sakai of Kagoshima University and Prof. S. Huzino of Kyushu University for helpful discussions and suggestions.

He would also like to express his sincere thanks to Prof. S. Hitotumatu of Kyoto University, Prof. M. Tanaka of Yamanashi University and Prof. T. Mitsui of Fukui University for their invaluable suggestions and advice. In the last, we would like to thank the referee for numerious fruitful comments.

\section{References}

[1] Byrne G, D. and Lambert, R, J., Pseudo-Runge-Kutta Methods involving two points, J. Assoc. Compt. Mach., 13 (1966), 114-123.

[2] Butcher, J. C., Coefficients for the study of Runge-Kutta integration Processes. $J$. Austral. Math. Soc., 3 (1963), 185-201.

[ 3 ] _-., Implicit Runge-Kutta Processes, Math Comp., 18 (1964), 50-64.

[4] Ceschino, F. and Kuntzmann, J. Numerical Solution of Initial value problems, PrenticeHall, Englewood Cliffs, New Jersey, 1966.

[5] Costabile, F., Methodi Pseudo-Runge-Kutta di seconda specie, Calcolo., 7 (1970), 305322.

[6] Henrici, P. Discrete Variable Methods in Ordinary Differential Equations, John Wiley \& Sons, New York, 1962.

[7] Heun, K., Neue Methode zur approximatven Integation der Differential gleichungen einer unabhangigen Veranderlichen, Z. Math. Physik., 45 (1900), 23-38.

[8] Kutta, W., Beitrag zur naherungsweizen Integration totaler Differential-gleichungen, Z. Math. Phys., 46 (1901), 435-453.

[9] Nakashima, M., On Pseudo-Runge-Kutta Methods with 2 and 3 stages, Publ. RIMS, Kyoto Univ., 18 (1982), 895-909.

[10] -., A modification of some Pseudo-Runge-Kutta Methods, to appear.

[11] ., On a Pseudo-Runge-Kutta Method of order 6, Proc. Japan Acad., 58 ser. A (1982), 66-68.

[12] Rosen, J. S. Multi-step Runge-Kutta methods. NASA Technical Note, NASA TN D4400 (1968).

[13] Runge, C., Uber die numerische Auflosung von Differentialgleichungen, Math, Ann., 46 (1895), 167-178.

[14] Tanaka, M., Pseudo-Runge-Kutta methods and their application to the estimation of truncation errors in 2nd and 3rd order Runge-Kutta methods, Joho Shori., 6 (1969), 406-417 (in Japanese).

[15] -. On the truncation errors of Runge-Kutta methods, Doctoral Thesis. Tokyo University. (1972). 